Article

\title{
Synthesis of ABA Tri-Block Co-Polymer Magnetopolymersomes via Electroporation for Potential Medical Application
}

\author{
Jennifer Bain, Matthew E. Berry ${ }^{\dagger}$, Catherine E. Dirks ${ }^{\dagger}$ and Sarah S. Staniland * \\ Received: 31 May 2015; Accepted: 17 November 2015; Published: 2 December 2015 \\ Academic Editor: Frank Wiesbrock \\ Department of Chemistry, University of Sheffield, Sheffield S3 7HF, UK; jbain1@sheffield.ac.uk (J.B.); \\ MBerry13@btinternet.com (M.E.B.); Catherine.dirks@hotmail.co.uk (C.E.D.) \\ * Correspondence: S.S.Staniland@sheffield.ac.uk; Tel.: +44-114-222-9539 \\ + These authors contributed equally to this work.
}

\begin{abstract}
The ABA tri-block copolymer poly(2-methyloxazoline)-poly(dimethylsiloxane)poly(2-methyloxazoline) (PMOXA-PDMS-PMOXA) is known for its capacity to mimic a bilayer membrane in that it is able to form vesicular polymersome structures. For this reason, it is the subject of extensive research and enables the development of more robust, adaptable and biocompatible alternatives to natural liposomes for biomedical applications. However, the poor solubility of this polymer renders published methods for forming vesicles unreproducible, hindering research and development of these polymersomes. Here we present an adapted, simpler method for the production of PMOXA-PDMS-PMOXA polymersomes of a narrow polydispersity (45 $\pm 5.8 \mathrm{~nm}$ ), via slow addition of aqueous solution to a new solvent/polymer mixture. We then magnetically functionalise these polymersomes to form magnetopolymersomes via in situ precipitation of iron-oxide magnetic nanoparticles (MNPs) within the PMOXA-PDMS-PMOXA polymersome core and membrane. This is achieved using electroporation to open pores within the membrane and to activate the formation of MNPs. The thick PMOXA-PDMS-PMOXA membrane is well known to be relatively non-permeable when compared to more commonly used di-block polymer membranes due a distinct difference in both size and chemistry and therefore very difficult to penetrate using standard biological methods. This paper presents for the first time the application of electroporation to an ABA tri-block polymersome membrane (PMOXA-PDMS-PMOXA) for intravesicular in situ precipitation of uniform MNPs $(2.6 \pm 0.5 \mathrm{~nm})$. The electroporation process facilitates the transport of MNP reactants across the membrane yielding in situ precipitation of MNPs. Further to differences in length and chemistry, a tri-block polymersome membrane structure differs from a natural lipid or di-block polymer membrane and as such the application and effects of electroporation on this type of polymersome is entirely novel. A mechanism is hypothesised to explain the final structure and composition of these biomedically applicable tri-block magnetopolymersomes.
\end{abstract}

Keywords: ABA tri-block co-polymer; polymersomes; magnetic nanoparticles; electroporation; biomedicine; bioinspired

\section{Introduction}

Vesicles are micro or nano scale chambers of solution, encapsulated by lipid (liposomes) or polymer (polymersomes). Liposomes specifically are ubiquitous in nature, serving numerous inter and intra cellular functions. Both liposomes and polymersomes are currently used in multiple industrial applications, particularly within the medical, food and cosmetics industries [1-3]. Perhaps the most prominent of these sectors, and with the greatest potential for application, is biomedicine, 
where vesicles have shown particular promise as drug delivery vehicles [4-6]. Vesicles can be used to encapsulate drugs and then later release them at the target location in response to specific stimuli by engineering of the vesicle membrane $[7,8]$. This targeted delivery means that lower doses of drugs can be administered, significantly improving the treatment efficiency whilst simultaneously reducing the magnitude and severity of any side effects. Currently, medical researchers are developing therapies that can simultaneously diagnose and treat a wide range of diseases, termed theranostic agents $[9,10]$. Theranostics are typically vesicles which incorporate therapeutics (via encapulation of a drug into a delivery vehicle) and diagonstic tools, such as fluorophores for optical imaging or magnetic nanoparticles (MNPs) for magnetic resonance imaging (MRI) [3,11-15]. The incorporation of MNPs means that vesicles can be used for magnetic targeted delivery of therapies, and allow monitoring via magnetic resonance imaging, as well as opening up treatment options such as magnetic hyperthermic ablation of tumours $[13,14]$.

Polymersomes are finding new applications across the entire field of biomedicine. The literature demonstrates that polymersomes can be engineered to incorporate a wide range of properties designed to meet the requirements of a specific application [15-17]. Polymersomes are already offering superior properties as drug delivery vehicles when compared with more traditional lipid vesicles. For example, polymer vesicles can have increased stability and low permeability, and properties such as increased molecular weight, makes them highly tunable. For instance, di-block polymer drug delivery carriers have been engineered to respond to stimuli including changes in; temperature, ionic strength, $\mathrm{pH}$ and even light (UV), all of which have huge potential for the successful release of cargo in many drug delivery applications [6-8,16,18,19]. pH can also control of the orientation of the polymer during assembly to determine which of the polymer blocks make up the outer leaflet of the polymersome [6,18]. The molecular weight of a polymer (of each block and also number of blocks) is fully controlled during its synthesis and has a direct effect on the hydrophobic thickness of the resulting membrane. This then subsequently impacts on the polymersome's stability, fluidity and diffusion properties $[1,3,16,20-22]$. Therefore it is entirely possible to adapt or engineer the polymer building blocks, and thus the resulting polymersome, to closer suit the needs of its eventual application. It is also possible to functionalise a polymersome membrane with a variety of biological moieties for drug delivery applications, as has been excellently outlined in the review by Pawar et al. [23]. These molecules include; antibodies, peptides, carbohydrates and small organic molecules; the choice of which is dependent upon the in vivo target. This functionalisation can be carried out by either covalent or non-covalent methods, depending on the chemistry of the polymer used to form the vesicle [23]. Reviewing this area of research highlights the high level of adaptability that polymersomes can offer, in terms of their susceptibility to engineering a wide range of functionalisation, and their potential for applications in biomedicine. This makes polymersomes an excellent material for use in the production of smarter nanomedical vehicles, as there is the ability to control the in vivo response, solubility, permeability, surface topology and lifetime of the vesicles in a tunable manner $[1,3]$.

\subsection{Poly(2-methyloxazoline)-poly(dimethylsiloxane)-poly(2-methyloxazoline) (PMOXA-PDMS-PMOXA) Polymersomes}

Many polymersomes, with varying composition and properties, have been explored for their drug delivery capabilities [22]. Most of these studies use AB di-block copolymers, resulting in di-block polymersomes becoming widely applied in biomedicine. However, ABA tri-block polymersomes may offer further advantages, particularly with respect to controlling membrane transport and encapsulation. Advantages of using a tri-block for compartmentalisation of a reaction include their low permeability $\left(5.7 \mu \mathrm{m} \cdot \mathrm{s}^{-1} \cdot \mathrm{bar}^{-1}\right.$ for a tri-block polymer compared to $0.22 \mu \mathrm{m} \cdot \mathrm{s}^{-1} \cdot \mathrm{bar}^{-1}$ for a di-block polymer) [24]. This lower permeability is due to an increased degree of polymerisation, and the distinctly different chemistries of each block when comparing the di- and tri-block polymers, such as the addition of end group reactivity in the case of 
PMOXA-PDMS-PMOXA. All of these factors contribute to the formation of a relatively impermeable polymer membrane (compared to the di-block polymer [24]). The key factor for this study is the monomeric nature of the resulting membrane, forming an amphiphilic monolayer as opposed to a more traditional bilayer, upon assembly. We predict that this, along with the increased block length will lead to significant differences in the rearrangement of the membrane to open pores. This change in chemistry, degree of polymerisation and membrane structure should give rise to better encapsulation and maintenance of the polymersome core contents (due to decreased permeability), along with the ability to stabilise and functionalise the vesicle by adaptation of reactive end groups. This functionality is particularly beneficial in biomedical applications, where confidence that the encapsulant will not easily leak out is paramount. Post formation functionalisation of the outer polymersome membrane can allow the attachment of drug and/or biomolecule therapies for targeted delivery. In this study we have chosen to explore the nanoreactor capabilities of the ABA tri-block polymer, PMOXA-PDMS-PMOXA.

A number of groups have studied this ABA tri-block polymer. Dinu et al. utilised the low permeability of the PMOXA-PDMS-PMOXA polymersome membrane to selectively polymerise the inner membrane exclusively [25]. PMOXA-PDMS-PMOXA has perhaps been most extensively studied by the group of Wolfgang Meier [26-30]. Their studies include the assessment of polymersome formation, controlling the permeability of the polymer vesicles, and the polymerisation of the individual block copolymers to form networks. Nardin et al. have demonstrated the use of PMOXA-PDMS-PMOXA vesicle synthetic membranes by successful incorporation of a functional enzyme into the vesicle for the potential improvement of genetic disorder treatments [27]. Specifically, the treatment of Mitochondrial Neurogastrointestinal Encephalomyopathy (MNGIE) by encapsulation of the enzyme using a nucleoside-specific porin (Tsx) has been described by Vocht et al. [31]. These studies have provided excellent proof of concept work for the use of PMOXA-PDMS-PMOXA polymersomes as vesicles for biomedicine. This also shows that PMOXA-PDMS-PMOXA is an ideal candidate for our investigation presented here, where we use an in situ MNP precipitation reaction to make magnetised polymersomes, and highlight their potential for use as magnetised biomedical vesicles.

PMOXA-PDMS-PMOXA polymersomes have been shown previously to be inherently biocompatible, which makes them excellent vehicles for future biomedical theranostic applications [3]. The presence of methacrylate groups at either end of the polymer (confirmed by NMR (Figure S1)), and gives rise to extensive polymerisation between individual copolymers via a chemical initiator, such as a photochemical reaction or irradiation with UV light [26]. On polymerisation of the methacrylate end groups, the polymer becomes covalently linked to neighbouring polymers, causing stretching of the polymer unit, in particular the hydrophobic region. This leads to a decrease in permeability and the eventual formation of extended structural networks within the vesicular membrane, making the polymersomes highly stable and robust (e.g., to changes in temperature) [29].

\subsection{Forming Magnetopolymersomes}

Combining MNPs with polymersomes to form magnetopolymersomes has so far been produced by loading pre-synthesised MNPs into a di-block co-polymer (such as PGA-b-PTMC) vesicle. These vesicles have also been loaded with anti-cancer drugs, and the resulting theranostic agent shows enhanced MRI contrast properties as well as successful magnetic targeting and drug delivery [32]. However, these promising magnetopolymersome biomedical agents are formed via a multistep process of crystallising and processing MNPs pre-vesicle formation, followed by their subsequent loading into polymersome [32], a process that is inefficient, expensive and time-consuming.

There are numerous ways to synthesise iron-oxide MNPs, many of which result in particles with differing sizes and shapes, which is highly undesirable [33]. Room temperature co-precipitation of iron-oxide MNPs (by addition of base to raise the $\mathrm{pH}$ of a mixed valence iron solution under 
an inert atmosphere) is simple, but results in a heterogeneous population of MNPs, which must be size filtered and magnetically separated before use. Methods that produce more mono-dispersed populations require more complex and costly synthesis and processing procedures. These often require high temperatures and toxic reagents, adding yet more synthetic steps to process MNPs before they can be considered suitable for biomedical application. Therefore a greener and milder synthetic route to making magnetically functionalised polymersomes with high quality mono-dispersed MNP populations remains an important challenge.

Bioinspired synthesis of magnetopolymersomes could enhance their properties and improve their function, particularly in the field of bio-nanomedicine. Nature performs precise reactions on the nanoscale by compartmentalising them within liposomes. Mimicking these systems has inspired the design of many small-scale synthetic reaction systems termed "nanoreactors" [34]. The concept of applying a biomimetic approach to the design and synthesis of a functional polymersome has been previously outlined both by our own group [34] and by Marguet et al. [35]. Here we take our inspiration from magnetotactic bacteria, which are able to perform controlled biomineralisation of magnetite MNPs within internal liposomes. Inmagnetotactic bacteria, iron ions cross the membrane into the vesicle interior via transmembrane iron-transport proteins, yielding the precipitation of magnetite within the vesicle core [36-38]. Here we adopt a similar strategy, where, for the first time, we use pre-formed $\mathrm{NaOH}$ filled (PMOXA-PDMS-PMOXA) vesicles as a nanoreactor for magnetite precipitation using electroporation to import iron ions.

Electroporation is a widely used technique in molecular biology to introduce DNA into a host cell. The application of an electric potential across a cell membrane causes temporary pores to form. This is because the electric field induces a transmembrane potential difference, which causes local defects that form pores. We have recently shown that this technique can be used for the inorganic precipitation of iron oxide nanoparticles in a nanoreactor vesicle arrangement [18]. This electroporation technique is also currently being explored as a method of getting drugs into delivery vesicles and for tumour ablation in vivo [39].

Although the mechanism of electroporation is largely unknown (mainly due to the short time scales and difficulty in probing pore formation), polymersome are believed to undergo similar poration to that observed in natural lipid cell membranes [40]. Furthermore, there are multiple parameters which can affect the outcome of electroporation. Most notable is the voltage applied across the membrane, but factors including path length of the cuvette, sample volume, temperature, pulse length and number of pulses can also be important. Studies of changes in phospholipid head group configuration via P-NMR, and observed changes in the vesicle structure has suggested possible mechanisms for electroporation [41]. Furthermore, molecular dynamics studies have offered multiple hypotheses regarding electroporation mechanisms [42-44]. However, it has not been possible to prove these hypotheses experimentally. There are two distinct theories on what is believed to happen when an electric potential is applied to a membrane. Both mechanisms propose that distortions in the membrane result in water penatration through a spontaeously formed unfavourable hydrophobic pore. The lipids then rearrange to form a stable more long-lived hydrophilic pore. This pore grows in size and is stabilised by a drop in field, causing a decrease in the conductance. The pore formation is then reversible and reseals over time, with the pore size, stability and/or lifetime being dependant on the surrounding pressure, the field strength and pulse time, as well as the membrane material [45]. The two mechanisms differ in how the distortions occur in the membrane.

The most widely accepted hypothesis; the transient aqueous pore formation mechanism, proposes an electric field-induced local distortion and rearrangment of the lipids due to conduction by the polar head groups. However, this has been disputed as electroporation is also effective in membranes that contain no polar (i.e., neutral) head groups. The alternative hypothesis: the water wire mechanism, involves alignment of the water/membrane interface, which undulates with the current. This results in a disortion, allowing water to pentatrate. However, it cannot be assumed that 
a tri-block polymer membrane will react to the application of an electric field in the same manner as that of a traditional bilayer.

We know from previous studied that pores can form in the PMOXA-PDMS-PMOXA 2D suspended membrane [29]. However, the difference in geometry and stability between a suspended membrane and a polymersome means that they are not entirely comparable. It is reasonable to believe that the generally accepted theories of electroporation, (both the transient aqueous pore mechanism [45-47] and the water wire mechanism [48]) could also be responsible for membrane pore formation in tri-block polymersomes. An ABA tri-block polymersome differs from previously studied di-block models significantly, in block length, monomer chemistry and the nature by which it forms a membrane. We believe that this study will both further advance the use of magnetopolymersomes in biomedicine and also help to inform the mechanism of electroporation in polymersomes.

\section{Experimental Section}

\subsection{Generation of PMOXA-PDMS-PMOXA Polymersomes}

All reagents were purchased from Sigma Aldrich, St. Louis, MO, USA. Control PMOXA-PDMS-PMOXA polymersomes were produced according to the method published by Nardin et al. [26] in the presence of excess ethanol as it was not possible to obtain the published $17 \% w / v$ of the polymer. Our new method of polymersome production consists of dissolving the PMOXA-PDMS-PMOXA polymer (5 mg, from Polymer Source Inc., Dorval (Montreal), QC, Canada) in a minimum volume of chloroform and adding this to a round bottom flask; containing a stirrer bar placed over a stirrer plate. $\mathrm{NaOH}(5 \mathrm{~mL}, 10 \mathrm{mM})$ is added to the polymer/solvent system using a Harvard Apparatus 11 Plus syringe pump driver at a rate of $5 \mu \mathrm{L} \cdot \mathrm{min}^{-1}$. On addition of $\mathrm{NaOH}$, the solution undergoes phase separation due to the presence of the solvent $\left(\mathrm{CHCl}_{3}\right)$. To enhance phase separation and remove polymer precipitate, the sample is transferred to a centrifuge and spun at 10,000 rpm, for $10 \mathrm{~min}$ in a Megafuge 40R (Thermo Scientific, Waltham, MA, USA) using a swinging bucket rotor (75003607, Thermo Scientific, Waltham, MA, USA). The aqueous layer containing PMOXA-PDMS-PMOXA polymersomes is isolated and cleaned up to remove excess $\mathrm{NaOH}$ solution using size exclusion chromatography (SEC) (PD-10 size exclusion column (GE Healthcare, Buckinghamshire, UK)). This is re-suspended in phosphate buffered saline (PBS) (NaCl $(8.0 \mathrm{~g}, 0.137 \mathrm{~mol}), \mathrm{KCl}\left(0.20 \mathrm{~g}, 2.68 \times 10^{-3} \mathrm{~mol}\right), \mathrm{Na}_{2} \mathrm{HPO}_{4} \cdot 2 \mathrm{H}_{2} \mathrm{O}\left(1.44 \mathrm{~g}, 8.09 \times 10^{-4} \mathrm{~mol}\right)$ and $\mathrm{KH}_{2} \mathrm{PO}_{4}\left(0.240 \mathrm{~g}, 1.76 \times 10^{-3} \mathrm{~mol}\right)$, dissolved in milliQ water $\left.(1 \mathrm{~L})\right)$ solution to maintain effective salt concentration across the polymer membrane.

\subsection{In Situ Synthesis of Magnetic Nanoparticles}

For the precipitation of the iron oxide nanoparticles within the ABA polymer membrane, solutions of $\mathrm{FeCl}_{2} \cdot 4 \mathrm{H}_{2} \mathrm{O}\left(1.98 \times 10^{-2} \mathrm{~g}, 1.00 \times 10^{-4} \mathrm{~mol}\right)$ and $\mathrm{FeCl}_{3} \cdot 6 \mathrm{H}_{2} \mathrm{O}\left(2.70 \times 10^{-2} \mathrm{~g}, 1.00 \times 10^{-4} \mathrm{~mol}\right)$ are each dissolved in degassed water $(10 \mathrm{~mL})$. The two solutions are then combined in the stoichiometric ratio for the precipitation of iron oxide, at a $v / v$ ratio of $1: 2 \mathrm{Fe}(\mathrm{II})$ : $\mathrm{Fe}(\mathrm{III})$. Polymersomes of PMOXA-PDMS-PMOXA are then added to the iron solution in a 1:1 $v / v$ ratio polymersome:iron solution. Polymersomes (at a concentration of $1 \mathrm{mg} \cdot \mathrm{mL}^{-1}$ ) are electroporated (using a Micropulser ${ }^{\mathrm{TM}}$, BioRad, Hertfordshire, UK) in aliquots of $200 \mu \mathrm{L}$, in a cuvette with a maximum volume of $800 \mu \mathrm{L}$. Cuvettes are cooled on ice before application of the voltage, and the solution is re-dispersed by shaking to prevent sedimentation. A pulse voltage of $750 \mathrm{~V}$ is applied to the sample (5 pulses, pulse length of $2 \mathrm{~ms}$ ). After application of all 5 pulses, magnetopolymersomes are cleaned up using SEC. MNP precipitation is confirmed by TEM imaging.

\subsection{Ion Transport by a Natural Ionophore}

Divalent cationic ionophore A23187 (Sigma Aldrich, St. Louis, MO, USA) is added to a solution of PMOXA-PDMS-PMOXA polymersomes encapsulating $10 \mathrm{mM} \mathrm{NaOH}$. The ionophore 
(used as provided) is added to a $10 \mathrm{mg} \cdot \mathrm{mL}^{-1}$ polymersome solution at a ratio of $1 \% \mathrm{v} / \mathrm{v}$ ionophore:polymersome solution. Iron solution identical to that used for electroporation is added to the sample, and incubated for 12-24 h. This should allow time for the ionophore to transport iron ions across the polymersome membrane to precipitate magnetic nanoparticles. Samples are imaged on TEM to look for the presence of iron oxide within the polymersome.

\subsection{Transmission Electron Microscopy}

Polymersomes (before and after electroporation) were imaged using a FEI Tecnai G2 Spirit transmission electron microscope. Before imaging, $5 \mu \mathrm{L}$ of the polymersome sample was spotted on to carbon coated copper EM grids. After incubation for approximately 2 min, excess sample was blotted off using filter paper, and the grids dried under vacuum. For non-electroporated polymersome samples, a $0.75 \%$ uranyl formate stain (to enhance polymersome contrast) was applied for $15 \mathrm{~s}$, before again blotting and drying under vacuum. Images were recorded using a Gatan $4000 \times 4000$ resolution charge coupled device (CCD) camera (Gatan, Pleasanton, CA, USA ), and processed using both Gatan micrograph and ImageJ software (National Institute of Health, Bethesda, MD, USA). Cryo-electron microscopy was performed on holey carbon grids prepared using $5 \mu \mathrm{L}$ of polymersome sample. The grids were subsequently blotted for $3 \mathrm{~s}$ (at 100\% humidity) and plunged into liquid ethane using a Vitrobot mark III. Samples were visualised on a Philips CM200, fitted with a Gatan $4000 \times 4000$ resolution CCD camera. All data were collected in low dose mode.

\subsection{Dynamic Light Scattering}

All measurements were recorded at $20{ }^{\circ} \mathrm{C}$ using a Zetasizer Nano series instrument (Malvern Instruments, Malvern, UK) equipped with a $4 \mathrm{~mW} 633 \mathrm{~nm}$ He-Ne laser and an avalanche photodiode detector. Polymer dispersions were diluted in deionised water to a concentration of $1.0 \% w / w$ and scattered light was detected at an angle of $173^{\circ}$.

\section{Results and Discussion}

Here we present a novel yet simple method for the production of stable and relatively monodisperse PMOXA-PDMS-PMOXA vesicles without the need for any post formation modifications such as extrusion. Previously, published methods [26] describe dissolving this tri-block polymer in ethanol solvent at a concentration of $17 \%$ by weight, followed by dilution to the critical aggregation concentration (cac). This published method could not be repeated in our lab, as we found that the polymer exhibited low solubility in such small solvent volumes, and was even poorly soluble when an excess volume of ethanol was added. Therefore, an entirely new route was designed, in which the polymer is dissolved in $\mathrm{CHCl}_{3}$ solvent at a final concentration above the cac of $0.15 \times 10^{-3} \mathrm{~g} \cdot \mathrm{mL}^{-1}$ in water. Addition of aqueous solution at a rate of $5 \mu \mathrm{L} \cdot \mathrm{min}^{-1}$ yields phase separation between the aqueous solution and the chloroform.

Isolation of the aqueous layer via centrifugation yields a solution of polymersomes with an average size of $45 \pm 5.8 \mathrm{~nm}$ from TEM images (Figure 1a,b), with the presence of small tubular structures (disregarded in the grainsizing). The corresponding DLS data show an average size of $32 \mathrm{~nm}$ for the polymersomes, and a polydispersity index of 0.5 (Figure 1b). This is in agreement with the TEM data, and yields a narrower dispersity than those observed in previous publications [26]. We compared our novel method with polymersomes prepared via the method developed by Nardin et al. [26] but with the use of excess ethanol (hence forth referred to as excess ethanol method). Rather than discrete, spherical polymersomes, TEM of the samples synthesised by this excess ethanol method [26] shows extensive branched dendritic polymer networks of approximately $1800 \mathrm{~nm}$ in length (Figure 1c), or $800 \mathrm{~nm}$ as measured by DLS (Figure 1d). It is probable that the improved, narrower dispersity obtained by our novel formation route is the result of the slow addition of the aqueous solution. It is evident from Figure 1c that, when the excess ethanol method [26] is used, extensive network polymerisation and/or subsequent aggregation is observed. We varied the rate 
of aqueous solution addition from between 5 and $50 \mu \mathrm{L} \cdot \mathrm{min}^{-1}$ and observed little difference in the morphology and size distribution of the polymersomes formed. However, when the aqueous solution is added rapidly to dilute the sample (i.e., as is specified in the excess ethanol method [26]), we observe the formation of large scale branched polymer networks (Figure 1c). Therefore, the rate of addition must be controlled to ensure that polymersomes rather than polymer networks are formed.

(a)

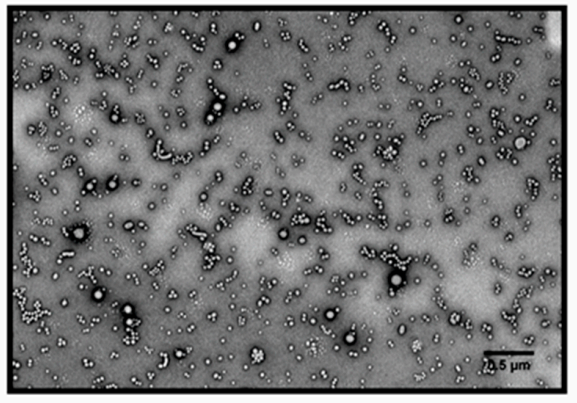

(b)

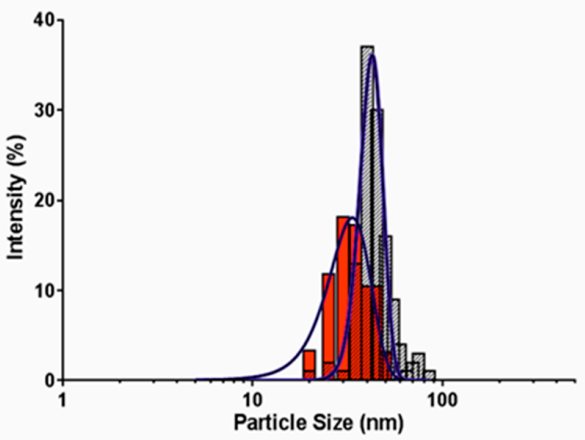

(c)

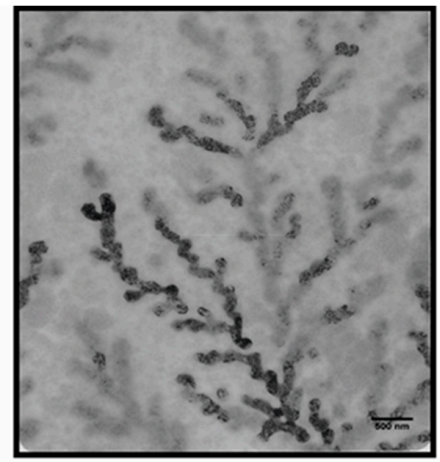

(d)

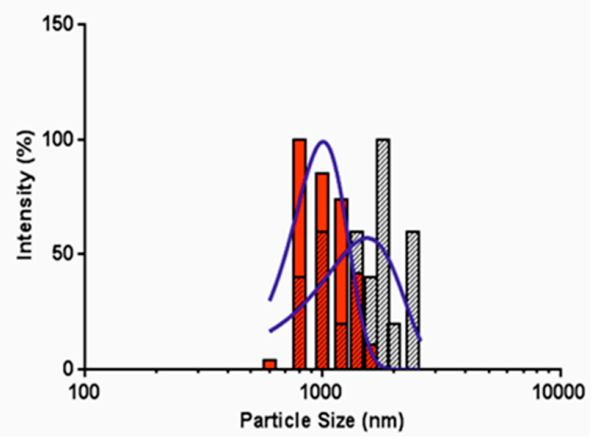

Figure 1. (a)Transmission electron microscopy (TEM) of poly(2-methyloxazoline)poly(dimethylsiloxane)-poly(2-methyloxazoline) (PMOXA-PDMS-PMOXA) polymersome sample produced via the adapted slow aqueous addition method; (b) comparison of TEM (Grey bars) grainsizing and Dynamic Light Scattering (DLS) (Red bars) sizing data show an average size of $45 \mathrm{~nm}$ compared to $32 \mathrm{~nm}$, respectively, and a polydispersity index of 0.5 ; (c) TEM of PMOXA-PDMS-PMOXA polymersomes produced via the previously published method with excess ethanol (excess ethanol method) [26] shows the presence of polymer networks and (d) the corresponding TEM (Grey bars) and DLS (Red Bars) grainsizing showing an average size of $1.8 \mu \mathrm{m}$ and $800 \mathrm{~nm}$ respectively.

The PMOXA-PDMS-PMOXA polymersomes synthesised by our novel slow addition method have been used as a nanoreactor for a room temperature co-precipitation reaction of magnetite MNPs within the polymersome membrane. $\mathrm{NaOH}(10 \mathrm{mM})$ is used as the aqueous solution for polymersome formation to create the high $\mathrm{pH}$ environment needed for magnetite precipitation within the vesicle core. This is followed by iron ion transport across the PMOXA-PDMS-PMOXA polymersome membrane. As discussed above, the successful encapsulation and use of these polymersomes as nanoreactors for other purposes has previously been shown by multiple groups $[25,28,29,31,49,50]$. In addition, it is believed that the PMOXA-PDMS-PMOXA membrane should behave in a manner analogous to a phospholipid membrane. However, the extended hydrophobic region of the polymer results in reduced permeability and a thicker membrane when compared to a biological lipid alternative [29]. Therefore, the incorporation of biological processes and biomembrane species into these tri-block polymersomes may be unlikely to succeed. As an alternative, we employ our recently published method of permeating a polymer membrane using electroporation [18]. Electroporation enhances membrane permeability, thus enabling the use of 
the polymersome as a nanoreactor for the precipitation MNPs within the polymersomes (Figure 2). Application of an electric potential across the membrane leads to poration, which allows the influx and efflux of the iron solution and $\mathrm{NaOH}$ magnetite precipitation reagents (respectively). On meeting in the membrane (as with our previous magnetopolymersome study [18]), iron oxide MNPs precipitate (Figure 2).
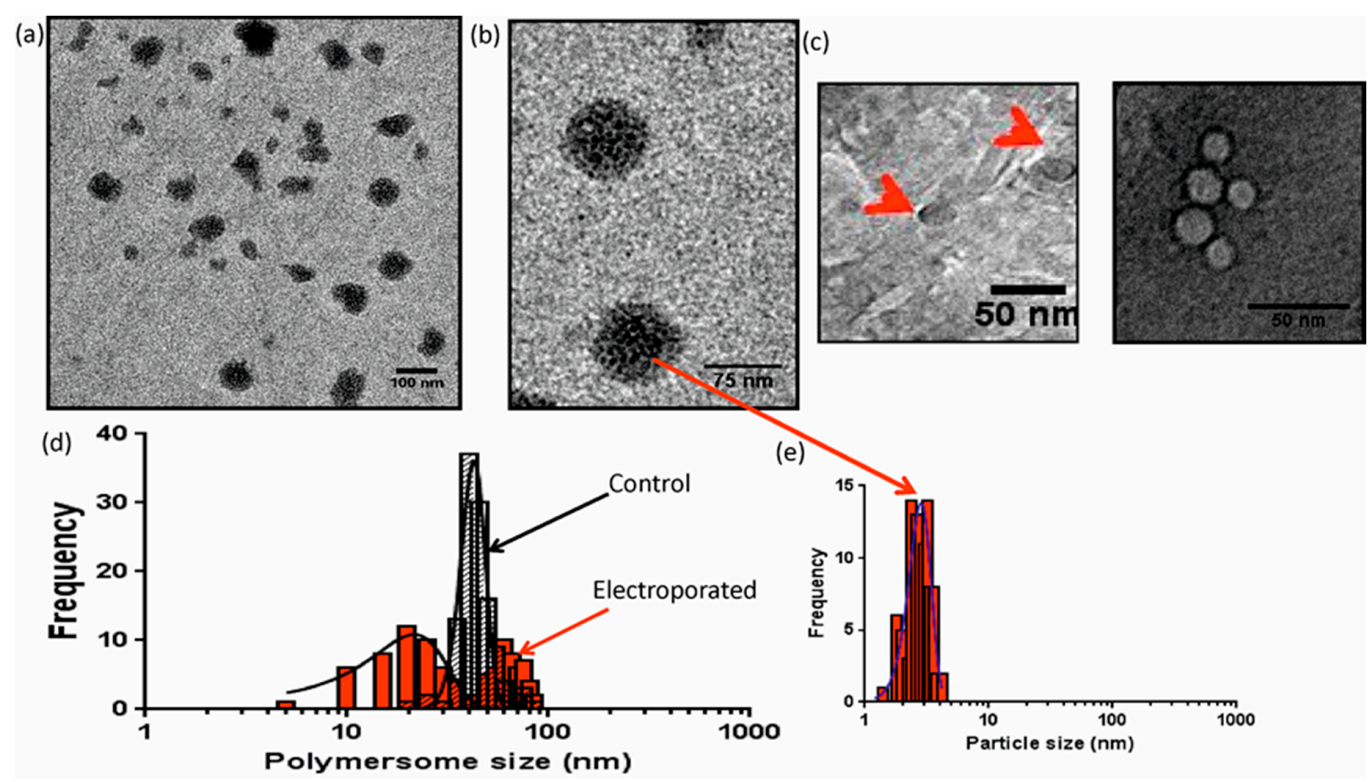

Figure 2. (a) The formation of iron oxide nanoparticles within tri-block PMOXA-PDMS-PMOXA polymersomes as the result of electroporation; (b) an example of a magnetopolymersome (at higher magnification) showing the distribution of nanoparticles within the polymersomes; (c) cryogenic TEM of magnetopolymersomes (indicated by red arrows in image) with electron density in both the polymersome core and within the membrane and of a size $(37 \pm 9 \mathrm{~nm})$ in agreement with the DLS; (d) the change in polymersome size before and after electroporation with unelectroporated control polymersomes (striped bars) with an average size of $45 \pm 8 \mathrm{~nm}$. After electroporation, a dual polymersome population at 20 and $60 \mathrm{~nm}$ is observed, giving an average polymersome size of $43.0 \pm 2.4 \mathrm{~nm}$; and (e) the corresponding MNP grainsizing from TEM, showing the average electroporated nanoparticle to be $2.6 \pm 0.9 \mathrm{~nm}$ in diameter. The magnetopolymersome were shown to be superparamagnetic by VSM analysis (Figure S4).

For comparison of transmembrane iron transport, a divalent cation ionophore (A23187) that is able to carry ferrous ions across a lipid bilayer membrane [51], was used to test if biological moieties are able to insert into and function within the PMOXA-PDMS-PMOXA membrane, and thus to transport iron ions into the polymersome core using conventional membrane transport. This proved to be unsuccessful (Figure S2), probably because the polymer membrane has an increased thickness when compared to a natural lipid membrane. In nature, A23187 would typically be used to span membranes of $4-5 \mathrm{~nm}$, which is about half the thickness of the PMOXA-PDMS-PMOXA membrane $(\sim 10 \mathrm{~nm})$. It is likely that this increased thickness of the polymers negatively impacts on the ionophore's transport capabilities, rendering it far less effective at transporting iron across polymer membranes when compared to thinner bi-layer lipid membranes. This aligns well with previous studies and suggests that natural methods of permeation are largely unsuccessful on these membranes.

The tri-block polymersomes are able to withstand voltages that are orders of magnitude higher than previously reported values for suspended membranes of PMOXA-PDMS-PMOXA. Seven hundred fifty volts were applied to the polymersomes five times (see Experimental Section). This is three to five times higher than a previously reported breakdown voltage of $1 \pm 0.2 \mathrm{~V}$ and 
$1.5 \pm 0.2 \mathrm{~V}$ for tri-block polymersomes following polymerisation [29]. Thus far, we have not observed breakdown of the polymersomes after the electric potential has been applied, as cryo-EM confirms both the size and morphology of the polymersome (Figure 2c) are maintained after electroporation. Post electroporation, tri-block polymersomes have an average size of $43.0 \pm 2.4 \mathrm{~nm}$, and only appear misshapen in regular TEM (i.e., when dried down rather than cryogenically frozen), which can be attributed to drying artefacts. The size of cryo-TEM imaged magnetopolymersomes is comparable to the un-electroporated control polymersomes shown in Figure 1a (i.e., $37 \pm 9 \mathrm{~nm}$ ).

On close inspection of the polymersomes (Figure 2), nanoparticles within the polymersome membrane can be seen. We are confident of the particles' tight association with the polymersomes, as they remain bound during the column clean-up process. When these polymersomes particles are compared to a room temperature co-precipitation of magnetite in the absence of polymersomes, much bigger, more polydisperse nanoparticles with an average size of $9.3 \pm 2.7 \mathrm{~nm}$ (Figure S3) are formed. The polymersome associated MNPs are smaller, with an average size of $2.6 \pm 0.9 \mathrm{~nm}$ (Figure 2e) and show improved monodispersity with regards to particle size and shape when compared to the room temperature co-precipitation. This distinct difference in particle size and dispersity is evidence that MNPs associated with the polymersomes have been precipitated in situ, giving rise to the tighter control observed. Furthermore it appears that the quantity of MNPs increases with their proximity to the core of the polymersome. TEM and Cryo-EM of the polymersomes confirms the MNPs position as being within the polymersome membrane (rather than being an aggregation of MNPs or just associated to the outside of the polymersome through electrostatic interactions) (Figure 2). Furthermore, the vesicle bilayer is clearly defined in the EM images obtained under cryogenic conditions.

Electroporated PMOXA-PDMS-PMOXA polymersomes are different to those formed with lipids and di-block co-polymers, due to the size, structural and chemical reasons discussed. We believe that this is the reason for an apparent increase in density of MNPs within the core of the vesicle, grading to fewer particles towards the edge of a vesicle. We thus believe there is a different mechanism in play in the mineralisation of the tri-block polymersomes when compared to the di-block and lipid bilayer vesicles. The core of the tri-block magnetopolymersomes appears to be denser when compared to our previous work using $A B$ di-block co-polymers [18]. It is likely that this increased density in the tri-block magnetopolymersomes is due to more iron ions being able to penetrate into the core of the polymersome during electroporation. This would result in some MNP precipitation within the tri-block polymersome lumen that is not observed in our study with the di-block co-polymer. This variance is most likely to be due to the difference in the composition (namely the degree of polymerisation and membrane structure) of the di- and tri-block polymers, and the effect that this has on the electroporation mechanism. It is possible that the tri-block vesicles are able to be stabilised during electroporation, possibly due to the difference in monomer chemistry between the two polymers, causing polymerisation of the methacrylate groups and stabilising pore formation. It is also a possibility that the increased length of the hydrophobic region of the polymer and consequently the decreased permeability reduces leakage of $\mathrm{NaOH}$ out of the membrane. This would lead to a higher concentration of reactant being present in the vesicle core during electroporation. The increased iron ion uptake observed suggests that the electroporation pore formation and molecular rearrangement of the membrane in a tri-block co-polymer differs from bilayer arrangements previously reported. Reasons for the difference in iron uptake could include the stabilisation of the pores due to polymerisation of the reactive end groups during electroporation. This would allow the formation of more particles in the core of the polymersome. This longer pore lifetime would allow for increased influx of iron ions across the membrane and a higher chance of reactants reaching the polymersome core giving rise to the increased MNP density observed in the polymersome core.

It has previously been proposed by both Lomora [50] and Itel [52] that when forming a membrane that tri-block polymers could completely double over on itself, in a configuration 
analogous to a "hairpin", which would render them equivalent to a di-block or lipid, thus assembling to form a membrane equivalent to a lipid bilayer (as shown by Figure 3b). However, in our polymer block proportions are 1700:4000:1700 g. $\mathrm{mol}^{-1}$, meaning the hydrophobic block length is more than double the hydrophilic block lengths. Therefore, further analysis (e.g., using computational modelling) would be necessary to determine if it is possible for the PMOXA-PDMS-PMOXA used in this study, to form a hairpin and behave analogous to a di-block membrane. Our hypothesis is that the membrane consists of one unit; a single polymer as opposed to two polymers forming a bilayer. This means that head group rearrangement to form a pore may be restricted (Figure 3). We propose a mechanism for rearrangement during electroporation, based on this property of PMOXA-PDMS-PMOXA. We suggest that, due to the flexibility within the tri-block polymer membrane, the introduction of polar water molecules into the membrane via electroporation causes the polymer to bend over on itself to shield the hydrophobic region of its structure (Figure 3c). This comes from both the length of the membrane and its high flexibility.

It is likely that adopting a "bent" configuration would be more entropically favourable than interactions between the PDMS and water. The increased electron density in the magnetopolymersome, suggesting increased iron oxide MNP precipitation, may be due to interactions between the reactive PMOXA end groups on the polymer (Figure 3c) maintaining the pores, or the extreme bending of the polymer increasing pore size and consequent iron ion influx. Either of these mechanisms could be the cause of the observed increase in the number of MNPs observed, as well as the greater MNP density in the core as the pores are retained for longer lifetimes. We aim to test this theory as part of a larger study of electroporation with tri-block co-polymers with a range of hydrophobic region lengths. We hypothesise that decreasing the hydrophobic region would reduce polymer flexibility, and thus the ability to shield the hydrophobic region. This would possibly result in less or no poration, with high fields resulting membrane breakdown.

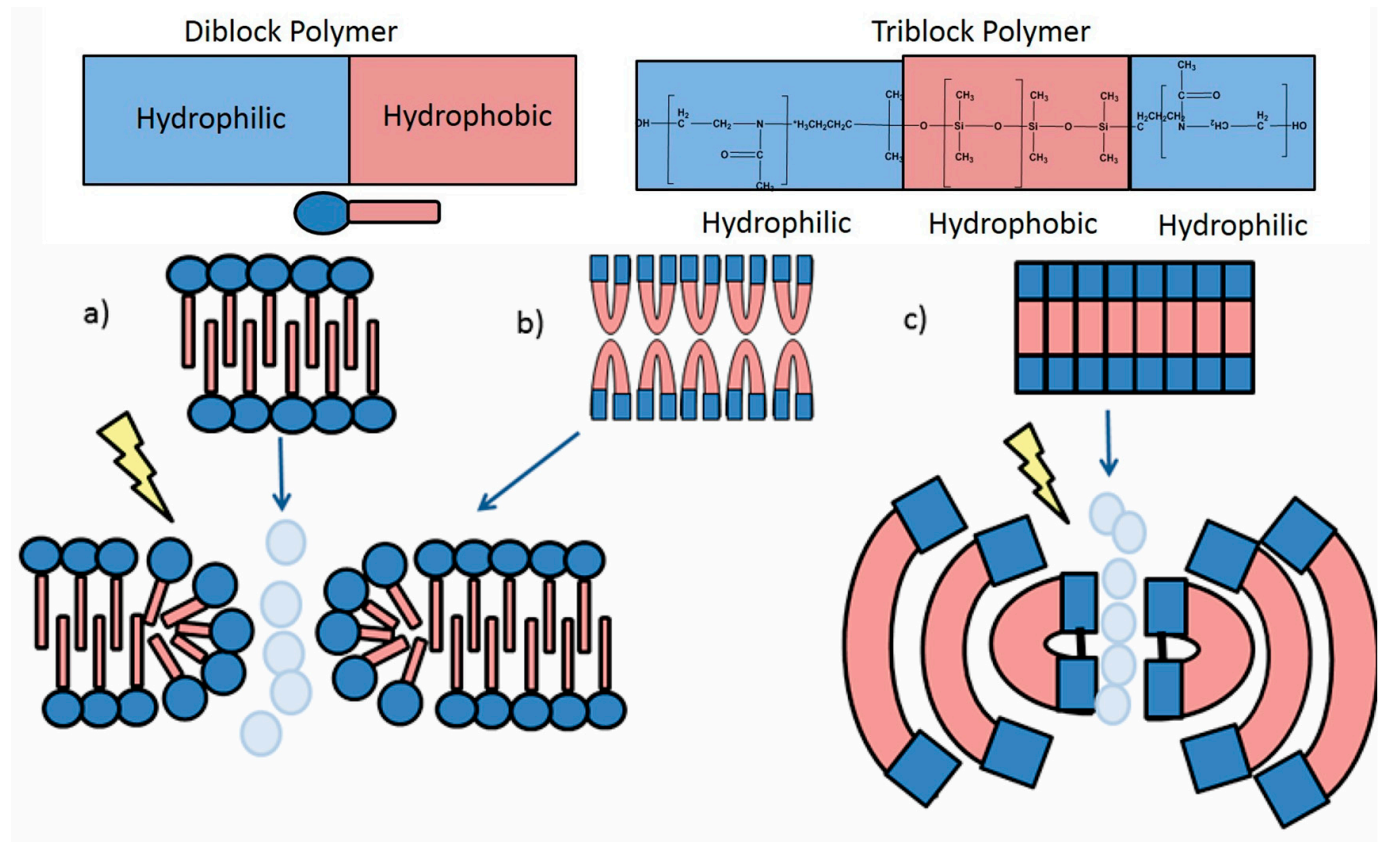

Figure 3. Schematic to show the hypothesis of the formation of pores by electroporation. (a) The case for a lipid or di-block polymers. The PMOXA-PDMS-PMOXA tri-block membrane could self-assemble in one of two ways, using electroporation (depicted by electric flash) to allow water (represented by blue droplets) to enter the bilayer; (b) A schematic of the folded over hairpin-like membrane form analogous to a more traditional bilayer, which can then follow the same mechanisms for electroporation as (a); and (c) the tri-block assembly as a monolayer. Upon electroporation, this bends over on it-self to shield the hydrophobic region of the structure. 


\section{Conclusions}

This paper presents a simplified method for the synthesis of monodisperse ABA tri-block polymersomes of PMOXA-PDMS-PMOXA. We have developed this method to produce stable, monodisperse vesicles without the need for any post formation modifications such as extrusion. We have demonstrated for the first time a method for synthetic permeation of a tri-block polymersome membrane by adaptation of our recently reported method of iron transport and iron oxide precipitation via electroporation [18]. This method is important, as it increases the permeability of the tri-block polymersome membranes, which is not as readily achieved using a cationic ionophore, due to the increased membrane thickness when compared to a natural lipid bilayer, making the incorporation of biological moieties difficult. It has long been thought that incorporation of biological molecules, such as ion channels and enzymes, are essential for the use of a polymersome as a nanoreactor and subsequently as biomedical agents. Our simplified electroporation method achieves greater permeation than that achieved with natural biomolecules (A23187 ionophore) whilst also offering more control than encapsulating reactants post formation.

Di-block magnetopolymersomes have shown increased diagnostic capabilities when applied to MRI imaging [18]. Electroporation of the polymer membrane for the in situ synthesis of iron oxide MNPs offer new vehicles for applications in biomedicine. The advantage of electroporation allows for the remote precipitation of magnetic nanoparticles. Using a polymer with reactive methacrylate end groups opens up potential for post formation functionalisation; this could offer a choice between core encapsulation of drugs during polymersome formation of post formation attachment to the outer surface of the polymersome, in this polymersome form.

We have observed increased MNP precipitation and demonstrated that the PMOXA-PDMS-PMOXA polymersomes can withstand electric potentials much higher than previously reported values for the supported membrane alone. The embedded MNPs are of a higher quality than standard room temperature co-precipitated MNPs, being both smaller (therefore exclusively superparamagnetic) and much more monodisperse. We present a new hypothesis for the mechanism of ABA membrane rearrangement during electroporation. This involves the bending of the individual polymers to shield the hydrophobic region. We suggest that this is specific to ABA membranes due to their increased flexibility and nature of their hydrophobic region. We believe this is the reason for the increased MNP precipitation we see when compared to di-block polymer magnetopolymersomes [18].

Supplementary Materials: Supplementary materials can be found at www.mdpi.com/2073-4360/ 7/12/1529/s1.

Acknowledgments: We would like to thank Svetomir Tsokov for assistance with TEM imaging and Johanna Galloway for consultation on the manuscript. We would also like to thank the EPSRC (Grant No. EP/I032355/2) for funding this research.

Author Contributions: The work was carried out by Catherine E. Dirks and Matthew E. Berry under the guidance of Jennifer Bain. All authors are members of the group of Sarah S. Staniland who designed the project. All TEM and data analysis was carried out by Jennifer Bain. Jennifer Bain and Sarah S. Staniland interpreted the data and wrote the manuscript.

Conflicts of Interest: The authors declare no conflict of interest.

\section{References}

1. Discher, D.E.; Ortiz, V.; Srinivas, G.; Klein, M.L.; Kim, Y.; Christian, D.; Cai, S.; Photos, P.; Ahmed, F. Emerging applications of polymersomes in delivery: From molecular dynamics to shrinkage of tumors. Prog. Polym. Sci. 2007, 32, 838-857. [CrossRef] [PubMed]

2. Discher, D.E.; Ahmed, F. Polymersomes. Annu. Rev. Biomed. Eng. 2006, 8, 323-341. [CrossRef] [PubMed] 
3. Brinkhuis, R.P.; Rutjes, F.P.; van Hest, J.C. Polymeric vesicles in biomedical applications. Polym. Chem. 2011, 2, 1449-1462. [CrossRef]

4. Ren, T.; Liu, Q.; Lu, H.; Liu, H.; Zhang, X.; Du, J. Multifunctional polymer vesicles for ultrasensitive magnetic resonance imaging and drug delivery. J. Mater.Chem. 2012, 22, 12329-12338. [CrossRef]

5. Abra, R.; Bankert, R.; Chen, F.; Egilmez, N.; Huang, K.; Saville, R.; Slater, J.; Sugano, M.; Yokota, S. The next generation of liposome delivery systems: Recent experience with tumor-targeted, sterically-stabilised immunoliposomes and active-loading gradients. J. Liposome Res. 2002, 12, 1-3. [CrossRef] [PubMed]

6. Lee, J.S.; Feijen, J. Polymersomes for drug delivery: Design, formation and characterisation. J. Control. Release 2012, 161, 473-483. [CrossRef] [PubMed]

7. Li, M.H.; Keller, P. Stimuli-responsive polymer vesicles. Soft Matter 2009, 5, 927-937. [CrossRef]

8. Ganta, S.; Devalapally, H.; Shahiwala, A.; Amiji, M. A review of stimuli-responsive nanocarriers for drug and gene delivery. J. Control. Release 2008, 126, 187-204. [CrossRef] [PubMed]

9. Santhosh, P.B.; Ulrih, N.P. Multifunctional superparamagnetic iron oxide nanoparticles: Promising tools in cancer theranostics. Cancer Lett. 2013, 336, 8-17. [CrossRef] [PubMed]

10. Svenson, S. Theranostics: Are we there yet? Mol. Pharm. 2013, 10, 848-856. [CrossRef] [PubMed]

11. Saha, K.; Agasti, S.S.; Kim, C.; Li, X.; Rotello, V.M. Gold nanoparticles in chemical and biological sensing. Chem. Rev. 2012, 112, 2739-2779. [CrossRef] [PubMed]

12. Zeng, S.; Yong, K.-T.; Roy, I.; Dinh, X.-Q.; Yu, X.; Luan, F. A review on functionalised gold nanoparticles for biosensing applications. Plasmonics 2011, 6, 491-506. [CrossRef]

13. Pankhurst, Q.; Thanh, N.; Jones, S.; Dobson, J. Progress in applications of magnetic nanoparticles in biomedicine. J. Phys. D Appl. Phys. 2009, 42, 224001. [CrossRef]

14. Pankhurst, Q.A.; Connolly, J.; Jones, S.; Dobson, J. Applications of magnetic nanoparticles in biomedicine. J. Phys. D Appl. Phys. 2003, 36, R167-R181. [CrossRef]

15. Thevenot, J.; Oliveira, H.; Lecommandoux, S. Polymersomes for theranostics. J. Drug Deliv. Sci. Technol. 2013, 23, 38-46. [CrossRef]

16. Massignani, M.; Lomas, H.; Battaglia, G. Polymersomes: A synthetic biological approach to encapsulation and delivery. Adv. Polym. Sci. 2010, 229, 115-154.

17. Meng, F.; Zhong, Z.; Feijen, J. Stimuli-responsive polymersomes for programmed drug delivery. Biomacromolecules 2009, 10, 197-209. [CrossRef] [PubMed]

18. Bain, J.; Ruiz-Pérez, L.; Kennerley, A.J.; Muench, S.P.; Thompson, R.; Battaglia, G.; Staniland, S.S. In situ formation of magnetopolymersomes via electroporation for mri. Sci. Rep. 2015, 5. [CrossRef] [PubMed]

19. Tong, X.; Wang, G.; Soldera, A.; Zhao, Y. How can azobenzene block copolymer vesicles be dissociated and reformed by light? J. Phys. Chem. B 2005, 109, 20281-20287. [CrossRef] [PubMed]

20. Discher, B.M.; Won, Y.-Y.; Ege, D.S.; Lee, J.C.-M.; Bates, F.S.; Discher, D.E.; Hammer, D.A. Polymersomes: Tough vesicles made from diblock copolymers. Science 1999, 284, 1143-1146. [CrossRef] [PubMed]

21. Blanazs, A.; Armes, S.P.; Ryan, A.J. Self-assembled block copolymer aggregates: From micelles to vesicles and their biological applications. Macromol. Rapid Commun. 2009, 30, 267-277. [CrossRef] [PubMed]

22. Prakash Jain, J.; Yenet Ayen, W.; Kumar, N. Self assembling polymers as polymersomes for drug delivery. Curr. Pharm. Des. 2011, 17, 65-79. [CrossRef]

23. Pawar, P.V.; Gohil, S.V.; Jain, J.P.; Kumar, N. Functionalised polymersomes for biomedical applications. Polym. Chem. 2013, 4, 3160-3176. [CrossRef]

24. Kumar, M.; Grzelakowski, M.; Zilles, J.; Clark, M.; Meier, W. Highly permeable polymeric membranes based on the incorporation of the functional water channel protein aquaporin Z. Proc. Natl. Acad. Sci. USA 2007, 104, 20719-20724. [CrossRef] [PubMed]

25. Dinu, M.V.; Spulber, M.; Renggli, K.; Wu, D.; Monnier, C.A.; Petri-Fink, A.; Bruns, N. Filling polymersomes with polymers by peroxidase-catalysed atom transfer radical polymerisation. Macromol. Rapid Commun. 2015, 36, 507-514. [CrossRef] [PubMed]

26. Nardin, C.; Hirt, T.; Leukel, J.; Meier, W. Polymerised ABA triblock copolymer vesicles. Langmuir 2000, 16, 1035-1041. [CrossRef]

27. Nardin, C.; Thoeni, S.; Widmer, J.; Winterhalter, M.; Meier, W. Nanoreactors based on (polymerized) ABA-triblock copolymer vesicles. Chem. Commun. 2000. [CrossRef] 
28. Meier, W.; Nardin, C.; Winterhalter, M. Reconstitution of channel proteins in (polymerised) ABA triblock copolymer membranes. Angew. Chem. Int. Ed. 2000, 39, 4599-4602. [CrossRef]

29. Nardin, C.; Winterhalter, M.; Meier, W. Giant free-standing aba triblock copolymer membranes. Langmuir 2000, 16, 7708-7712. [CrossRef]

30. Nardin, C.; Widmer, J.; Winterhalter, M.; Meier, W. Amphiphilic block copolymer nanocontainers as bioreactors. Eur. Phys. J. E 2001, 4, 403-410. [CrossRef]

31. De Vocht, C.; Ranquin, A.; Willaert, R.; van Ginderachter, J.A.; Vanhaecke, T.; Rogiers, V.; Versées, W.; van Gelder, P.; Steyaert, J. Assessment of stability, toxicity and immunogenicity of new polymeric nanoreactors for use in enzyme replacement therapy of mngie. J. Control. Release 2009, 137, 246-254. [CrossRef] [PubMed]

32. Sanson, C.; Schatz, C.; le Meins, J.-F.O.; Brûlet, A.; Soum, A.; Lecommandoux, S.B. Biocompatible and biodegradable poly(trimethylene carbonate)- $b$-poly(L-glutamic acid) polymersomes: Size control and stability. Langmuir 2009, 26, 2751-2760. [CrossRef] [PubMed]

33. Laurent, S.; Bridot, J.L.; vander Elst, L.; Muller, R.N. Magnetic iron oxide nanoparticles for biomedical applications. Future 2010, 2, 427-449. [CrossRef] [PubMed]

34. Staniland, S.S.; Bain, J. Bioinspired nanoreactors for the biomineralisation of metallic-based nanoparticles for nanomedicine. Phys. Chem. Chem. Phys. 2015, 17, 15508-15521.

35. Marguet, M.; Bonduelle, C.; Lecommandoux, S. Multicompartmentalised polymeric systems: Towards biomimetic cellular structure and function. Chem. Soc. Rev. 2013, 42, 512-529. [CrossRef] [PubMed]

36. Nies, D.H. How iron is transported into magnetosomes. Mol. Microbiol. 2011, 82, 792-796. [CrossRef] [PubMed]

37. Komeili, A.; Li, Z.; Newman, D.K.; Jensen, G.J. Magnetosomes are cell membrane invaginations organised by the actin-like protein mamk. Science 2006, 311, 242-245. [CrossRef] [PubMed]

38. Bazylinski, D.A.; Schubbe, S. Controlled biomineralisation by and applications of magnetotactic bacteria. Adv. Appl. Microbiol. 2007, 62, 21-62. [PubMed]

39. Garcia, P.; Rossmeisl, J., Jr.; Neal, R., II; Ellis, T.; Olson, J.; Henao-Guerrero, N.; Robertson, J.; Davalos, R. Intracranial nonthermal irreversible electroporation: In vivo analysis. J. Membr. Biol. 2010, 236, 127-136. [CrossRef] [PubMed]

40. Wang, L.; Chierico, L.; Little, D.; Patikarnmonthon, N.; Yang, Z.; Azzouz, M.; Madsen, J.; Armes, S.P.; Battaglia, G. Encapsulation of biomacromolecules within polymersomes by electroporation. Angew. Chem. Int. Ed. 2012, 51, 11122-11125. [CrossRef] [PubMed]

41. Lopez, A.; Rols, M.; Teissie, J. Phosphorus-31 NMR analysis of membrane phospholipid organisation in viable, reversibly electropermeabilised chinese hamster ovary cells. Biochemistry 1988, 27, 1222-1228. [CrossRef] [PubMed]

42. Tieleman, D.P.; Leontiadou, H.; Mark, A.E.; Marrink, S.-J. Simulation of pore formation in lipid bilayers by mechanical stress and electric fields. J. Am. Chem. Soc. 2003, 125, 6382-6383. [CrossRef] [PubMed]

43. Tieleman, D.P. The molecular basis of electroporation. BMC Biochem. 2004, 5, 10. [CrossRef] [PubMed]

44. Bennett, W.D.; Sapay, N.; Tieleman, D.P. Atomistic simulations of pore formation and closure in lipid bilayers. Biophys. J. 2014, 106, 210-219. [CrossRef] [PubMed]

45. Weaver, J.C.; Chizmadzhev, Y.A. Theory of electroporation: A review. Bioelectrochem. Bioenerg. 1996, 41, 135-160. [CrossRef]

46. Powell, K.T.; Weaver, J.C. Transient aqueous pores in bilayer membranes: A statistical theory. Bioelectrochem. Bioenerg. 1986, 15, 211-227. [CrossRef]

47. Weaver, J.C. Electroporation: A general phenomenon for manipulating cells and tissues. J. Cell. Biochem. 1993, 51, 426-435. [CrossRef] [PubMed]

48. Melikov, K.C.; Frolov, V.A.; Shcherbakov, A.; Samsonov, A.V.; Chizmadzhev, Y.A.; Chernomordik, L.V. Voltage-induced nonconductive pre-pores and metastable single pores in unmodified planar lipid bilayer. Biophys. J. 2001, 80, 1829-1836. [CrossRef]

49. Krishnamoorthy, B.; Karanam, V.; Chellan, V.R.; Siram, K.; Natarajan, T.S.; Gregory, M. Polymersomes as an effective drug delivery system for glioma-A review. J. Drug Target. 2014, 22, 469-477. [CrossRef] [PubMed]

50. Lomora, M.; Itel, F.; Dinu, I.A.; Palivan, C.G. Selective ion-permeable membranes by insertion of biopores into polymersomes. Phys. Chem. Chem. Phys. 2015, 28, 15538-15546. [CrossRef] [PubMed] 
51. Reed, P.W.; Lardy, H.A. A23187: A divalent cation ionophore. J. Biol. Chem. 1972, 247, 6970-6977. [PubMed] 52. Itel, F.; Chami, M.; Najer, A.; Lörcher, S.; Wu, D.; Dinu, I.A.; Meier, W. Molecular organisation and dynamics in polymersome membranes: A lateral diffusion study. Macromolecules 2014, 47, 7588-7596. [CrossRef]

(c) 2015 by the authors; licensee MDPI, Basel, Switzerland. This article is an open access article distributed under the terms and conditions of the Creative Commons by Attribution (CC-BY) license (http://creativecommons.org/licenses/by/4.0/). 